\title{
ASTR.0NOMISCHE NACHRICHTEN.
}

\section{Observations de Jupiter dans l'opposition de 1898. Par F. Comas Sold.}

[Avec une planche].

Quoique le temps a été ici extrêmement défavorable pendant cette opposition, j'ai pu faire un nombre suffisant d'observations pour permettre une description assez détaillée des principales particularités que la planète a offertes pendant ce temps. Ces observations sont commencées le 18 Janvier et terminées le 12 Juin 1898 .

L'aspect général de Jupiter n'a pas souffert de grands changements. Tout semble indiquer que la planète se trouvait dans une période de calme relative. Cette tendance a été plus manifeste que partout dans la bande équatoriale boréale, qui était l'année dernière très large, double et parfois triple, tandis qu'elle a été vue maintenant simple, montrant une structure assez uniforme et un ton généralement très foncé et rougeâtre; ses bords ont été toujours bien tranchés. Cependant on pouvait voir quelques restes de la composante boréale de cette bande sous l'apparence, principalement, de taches foncées, souvent allongées et étroites.

Dans une communication à Astr. Nachr. * (A.N.3510), j'ai fait connaître mes observations sur ces taches jusqu'au I Mai. J'ajouterai que la tache $\Pi$, qui était invisible le I 9 Avril et les jours suivants, j'ai pu la revoir le 6 Juin avec une très belle image. Elle était très petite et difficile d voir; sa longitude était de $238: 5$.

Pour les autres deux taches $\mathrm{j}$ 'ai trouvé les suivants résultats qui complètent ceux que j'ai communiqué déjà (système II de M. Crommelin).

$\begin{array}{crc}\text { Tache I } & 3 \text { Juin } & \lambda \\ \text {, III } & 2089 \text { Mai } & 284.9 \\ \text {, } & 6 \text { Juin } & 280.2\end{array}$

En comparant toutes mes longitudes des trois taches avec celles des premières observations des mêmes faites par MM. Fauth et Brenner (A. N. 3476, 3488), je trouve, ayant compte du poids de chaque résultat dans le calcul des moyennes, les suivants mouvements par jour, vers l'Ouest:

$$
\begin{array}{ccc}
\text { Tache I } & 0.28 \\
\text {, II } & 0.34 \\
\text { III } & 0.42
\end{array}
$$

Ce qui donne respectivement pour la durée de rotation:

$$
9^{\mathrm{h}} 55^{\mathrm{m}} 29^{\mathrm{s}} \mathrm{I} ; \quad 9^{\mathrm{h}} 55^{\mathrm{m}} 26^{\mathrm{s}} \cdot 7 ; \quad 9^{\mathrm{h}} 55^{\mathrm{m}} 23^{\mathrm{s}} \cdot 4 \text {. }
$$

Je fais omission d'autres détails concernant cette région de la planète qui se trouvent décrits dans la communication citée. Dans le planisphère adjoint sont repré- sentées les trois taches dans la longitude correspondante au jour de l'opposition.

La bande temperée boréale a été très difficile et la plupart des fois impossible à voir. En général, il y a eu très peu de détails dans la calotte boréale. On y voyait seulement quelque bande irrégulière et fugitive. Le $3 \mathrm{Mai}$, avec une image parfaite, on ne voyait, a partir de la bande équatoriale boréale, aucun détail: on remarquait seulement que le ton grisâtre, qui dominait dans cette calotte, devenait de plus en plus sombre vers le pole boréal.

D'ordinaire, la zone equatoriale n'a pas été riche de détails. Ce qui a offert de plus notable a été, sans doute, son intense coloration jaune rougeâtre ou orange jaunâtre; cette coloration a diminué beaucoup vers la fin des observations, mais dernièrement elle a été de nouveau très accusée. Comme d'habitude, cette zone a été traversée plus ou moins obliquement et dans tous les sens par des trainées grises qui sortaient des taches foncées du bord boréal de la bande équatoriale australe. Parmi ces trainées et en contact avec elles, ont été souvent visibles des taches blanches diffuses, en général de grandes dimensions. Quelques unes de ces taches blanches sont, sans doute, plus élevées que les autres détails de la zone équatoriale, puisque plusieurs fois j'ai observé qu'elles se détachaient avec un grand éclat près des bords de la planète, tandis qu'elles étaient peu visibles vers le méridien central, phénomène semblable à celui que l'on observe dans les facules solaires. Le mauvais temps presque continuel que nous avons eu ici pendant cette année m'a empêché de faire des observations quelque peu complètes sur la durée de rotation de ces taches; mais il est évident que celles-ci, du même que certaines taches foncées du bord $\mathbf{N}$ de la bande équatoriale australe, ont en général un rapide mouvement de rotation, comme l'a montré $M$. Denning et d'autres astronomes.

Le I3 Avril, avec une superbe image, on voyait facilement que la zone équatoriale était floconneuse, formée de grandes taches foncées et arrondies, comme montre le planisphère vers la longitude de $70^{\circ}$. Avec des bonnes images était parfois visible, quoique difficilement, une étroite bande équatoriale, et le $9 \mathrm{Mai}$ on pouvait voir avec attention un filet brillant très étroit en contact avec la bande équatoriale boréale.

La grande bande équatoriale australe a offert l'aspect d'habitude: foncée, rougeâtre, double et peuplée de nombreuses taches très sombres, surtout vers le bord boréal. 
Le planisphère donne une idée claire de l'aspect de cette bande en différentes époques è régions. Il faut remarquer cependant l'aspect de cette bande à gauche de la tache rouge. Comme on peut voir, la bande est claire à l'Ouest, mais à partir de la tache ou région blanche qui se trouve dans la composante boréale $\left(355^{\circ}\right.$ de longitude), elle prend subitement une intensité que je n'avais vue jamais pendant les ro années de mon observation continue de Jupiter. On dirai que la matière constitutive de la bande trouvait, dans la tache blanche citée, une résistance enorme au mouvement vers l'Ouest.

La coloration des taches foncées, rondes et elliptiques, du bord boréal de cette bande n'était pas rouge, mais plutôt verdâtre et bleuâtre.

La tache rouge était toujours très pâle et grise, mais malgré sa grande faiblesse j'ai pu voir, par de bonnes images, tout son contour elliptique. Sa pointe orientale était presque toujours plus foncée que le reste; quelquefois on pouvait yoir une petite tache sombre dans cet endroit.

Mes observations de passages de la pointe orientale par le méridien central ont donné les suivants résultats:

\begin{tabular}{|c|c|c|}
\hline $\begin{array}{r}20 \\
9 \\
7\end{array}$ & $\begin{array}{l}\text { 0 Avril } \\
9 \text { Mai } \\
7 \text { Juin }\end{array}$ & $\begin{array}{c}\lambda \\
35^{\circ} \cdot 9 \\
39.6 \\
34.2\end{array}$ \\
\hline Moyenne 23 & $3 \mathrm{Maj}$ & 36.6 \\
\hline
\end{tabular}

La zone claire qui suit au $S$ de la grande bande équatoriale australe a présenté un grand nombre de taches blanches en contact avec la bande équatoriale australe. Quelques unes de ces taches étaient de grandes dimensions, circulaires, brillantes et bien tranchees; une d'elles montrait au travers, comme par un effet de transparence, la grande bande (longitude $=5^{\circ}$ ) en la recouvrant un peu (probablemunt ces taches se trouvent à un niveau plus haut que la bande équatoriale). Mais la plupart des mêmes étaient moutonnées, en contact avec la bande équatoriale, constituant une zone brillante et floconneuse.
Aucune de ces taches s'est projettée sur la tache rouge; mais j'ai pu voir à plusieurs reprises quelques unes dans l'espace compris entre la tache rouge et l'inflexion de la bande équatoriale australe (voyez le planisphère). L'identification très difficile de ces taches blanches n'a empêché d'obtenir des résultats quelque peu sûrs sur la durée de la rotation.

La bande tempérée australe a été floconneuse, irrégulière et quelques fois double, quoique ce dédoublement fut toujours très difficile. De plus elle a été variable, dans quelques régions intense. et dans d'autres assez faible. On y voyait aussi des petites taches foncées, sans que la bande présentait aucun maximum d'intensité sur la tache rouge, comme c'était d'habitude les années antérieures.

Plus au $S$ suit une autre bande très variable; parfois très intense et presque toujours floconneuse. Ces deux dernières bandes ont été séparées, en général, par une région brillante offrant souvent, avec toute évidence, l'aspect de grandes taches blanches. Plus au $\mathrm{S}$ encore que la bande plus australe citée, $j$ 'ai vu aussi parfaitement, malgré sa haute latitude, des taches blanches. La calotte polaire australe a été grise verdâtre.

Je peux ajouter, en terminant, que plus l'image était bonne plus on voyait partout la constitution granulaire des details de Jupiter; c'est à dire des taches rondes, brillantes ou foncées, alignées parallèlement a l'équateur jovien et souvent groupées en familles ou formant des alignements isolés. L'examen seul du planisphère ci-joint montre bien ces faits généraux qui sont, sans doute, intimement liés avec le procès de formation de la plupart des détails que nous observons dans le disque de Jupiter.

Les latitudes des principales bandes d'après mes mesures micrométriques ont été inscrites dans la communication citée à Astr. Nachr.s (A. N. 3510 ). Dans le planisphère, ces positions sont graphiquement représentées.

Instrument employé dans toutes ces observations: Equatorial double de $22 \mathrm{~cm}$ avec des grossissements variant depuis 100 fois jusqu'd 260 ; le plus souvent 180 .

Observatori Català, Sant Feliu de Guíxols, Espagne, is Juin 1898.

F. Comas Sold.

\section{Note on some of the variable stars of the cluster Messier 5 .}

From observations with the 40 inch Refractor of the Yerkes Observatory.

\section{By E. E. Barnard.}

I was very much interested in the discovery by photography of a great number of variable stars in some of the globular clusters - such as $\infty$ Centauri, M. 3 , M. 5 , etc. by Professor Bailey at the Arequipa station of the Harvard College Observatory. As I had decided to take up the measurement of the stars in some of these clusters with the great telescope, it was evident that while doing this work there would be an excellent opportunity to visually verify the variability of a few of these small stars for which the 40 inch would be specially suitable. As the first work was to be in Messier 5, Professor E. C. Pickering, at my request, kindly sent me a photograph of the cluster with the variables marked upon it. I am also indebted to Professor Pickering for other courtesies during this work.

As it seemed an unnecessary and useless labor to attempt the verification of all these variables, I selected at random several of the stars but refrained from any knowledge of their periods.

Since last December a large number of observations of the brightness of these stars has been accumulated. I cannot now take the time to properly discuss this mass of material. It has however appeared to me that a few brief remarks on the results of the work may be interesting as 